\title{
Running couplings and triviality of field theories on non-commutative spaces
}

\author{
Emil T. Akhmedov, Philip DeBoer and Gordon W. Semenoff \\ Department of Physics and Astronomy, \\ University of British Columbia, \\ Vancouver, British Columbia, Canada V6T 1 Z1.
}

November 2, 2018

\begin{abstract}
We examine the issue of renormalizability of asymtotically free field theories on non-commutative spaces. As an example, we solve the non-commutative $\mathrm{O}(\mathrm{N})$ invariant Gross-Neveu model at large N. On commutative space this is a renormalizable model with non-trivial interactions. On the noncommutative space, if we take the translation invariant ground state, we find that the model is nonrenormalizable. Removing the ultraviolet cutoff yields a trivial non-interacting theory.
\end{abstract}

\footnotetext{
${ }^{*}$ Permanent Address: Institute of Theoretical and Experimental Physics, B. Cheremushkinskaya 25, 117259 Moscow, Russia
} 
1. Motivated by the fact that they arise as the low energy limits of string theories with antisymmetric tensor backgrounds [1, 2, 3, 4, 5, 6, field theories on non-commutative spaces have recently received a great deal of attention. On non-commutative $R^{d}$, the coordinates have the algebra $\left[x^{\mu}, x^{\nu}\right]=-i \theta^{\mu \nu}$, where $\theta$ is an antisymmetric matrix. This noncommutativity can also be implemented by replacing the usual product for multiplying functions by the associative, noncommutative and non-local $*$-product,

$$
f(x) * g(x) \equiv \lim _{y \rightarrow x} \exp \left(-\frac{i}{2} \theta^{\mu \nu} \frac{\partial}{\partial x^{\mu}} \frac{\partial}{\partial y^{\nu}}\right) f(x) g(y)
$$

When non-commutative field theories are obtained as limits of string theory, the massive string excitations decouple. For this reason the non-commutative field theories are believed to be unitary and renormalizable. In fact, for some theories, unitarity has been denomstrated explicitly at one-loop order [7]. This raises the interesting possibility that there exist consistent, unitary, and nontrivial nonlocal field theories. In this Letter, we examine this issue in the nonperturbative context of a solvable model.

There are several features of non-commutative field theories which distinguish them from their commutative analogs. One already occurs in scalar field theory. The non-commutativity affects the spectrum and interactions of the theory at low energy scales, below the momentum scale $1 / \sqrt{|\theta|}$ set by the dimensional parameter $\theta_{\mu \nu}$ and in fact below the mass scales of the particles already in the model. This has been associated with the phenomenon of ultraviolet/infrared (UV/IR) duality familiar from the behavior of D-branes in string theory [8, 9].

In non-commutative field theory, there is a sense in which perturbative contributions to a given diagram can be divided into planar and non-planar graphs [10]. The Feynman integrands of planar graphs are as they were in the commutative case. The integrands of non-planar graphs are modified by phases containing external and internal loop momenta. The presence of these phases improves the high-momentum behavior of Feynman integrals. The most dramatic effect occurs in diagrams which are ultraviolet divergent. Planar diagrams diverge and must be defined using a high momentum cutoff, $\Lambda$. The non-planar diagrams generally converge, the ultraviolet cutoff being replaced by $\Lambda_{\text {eff }}(p)=\Lambda / \sqrt{1+\Lambda^{2}(\theta p)^{2}}$. For any non-zero momentum, this effective cutoff has a finite limit, $\Lambda_{\text {eff }} \sim 1 /|\theta p|$, as $\Lambda \rightarrow \infty$.

For example, at one loop order in 4-dimensional $\phi^{4}$-theory, the radiative correction to the scalar self-energy in the commutative version is a quadratically divergent constant. In the non-commutative theory, there are two contributions, a planar and non-planar one. The non-planar one turns out to be a function of external momentum. At small momentum, [8, 9]

$$
\Gamma^{(2)}(p)=\frac{g^{2}}{48 \pi^{2}}\left(\Lambda^{2}-m^{2} \ln \frac{\Lambda^{2}}{m^{2}}\right)+\frac{g^{2}}{96 \pi^{2}}\left(\Lambda_{\mathrm{eff}}^{2}(p)-m^{2} \ln \frac{\Lambda_{\mathrm{eff}}^{2}(p)}{m^{2}}\right)+\ldots
$$

Here, $m$ is the scalar field mass and $g^{2}$ is the dimensionless $\phi^{4}$ coupling constant. The second term has a pole at very low momenta. It also has a logarithmic 
cut singularity at small momenta. These have been argued to arise from new degrees of freedom with exotic dispersion relations or perhaps propagating in higher dimensions [9]. They have also been argued to lead to exotic translation non-invariant "striped" phases of scalar field theory [11.

Another place where ultraviolet divergences typically occur and have interesting effects is in the renormalization of dimensionless coupling constants. The leading corrections to the coupling constant in 4-dimensional $\phi^{4}$-theory are logarithmically divergent. The small momentum limit of the 4-point function was computed in [8] as

$$
\begin{aligned}
\Gamma^{(4)}(p, q, r, s)=g^{2}- & \frac{g^{2}}{2 \cdot 2^{5} \pi^{2}}\left\{2 \ln \frac{\Lambda^{2}}{m^{2}}+\ln \frac{1}{m^{2}(\theta p)^{2}}+\ln \frac{1}{m^{2}(\theta q)^{2}}+\right. \\
+ & \ln \frac{1}{m^{2}(\theta r)^{2}}+\ln \frac{1}{m^{2}(\theta s)^{2}}+\ln \frac{1}{m^{2}(\theta(q+r))^{2}}+ \\
& \left.+\ln \frac{1}{m^{2}(\theta(q+s))^{2}}+\ln \frac{1}{m^{2}(\theta(r+s))^{2}}\right\}+\ldots
\end{aligned}
$$

$(p+q+r+s=0)$ The first contribution is from planar diagrams and the others are from non-planar diagrams and they depend explicitly on the parameter $\theta$. What is remarkable about (3) is that, in spite of the mass gap, the effective coupling constant is logarithmically singular at small momentum, similar to its large momentum limit but with $p^{2} / \Lambda^{2}$ replaced by $m^{2}(\theta p)^{2}$. If we were to sum the leading logarithmically singular diagrams to all orders, we would obtain a coupling constant which runs at small momentum scales. This is distinct from the behavior of commutative field theory where the running of coupling constants is cutoff by mass scales. For example, in quantum electrodynamics which, like $\phi^{4}$-theory is infrared free, the coupling constant runs at energies much larger than the electron mass, but as the energy is lowered, it freezes at the value $e^{2} / 4 \pi \sim 1 / 137$ and is the same at all lower energy scales. In a noncommutative theory, it appears that masses do not cutoff the running of coupling constants. We note that this is a non-perturbative issue, which occurs in addition to the perturbative renormalizability of non-commutative field theories which has recently been examined in detail [12, 13, 14].

Of particular interest are asymptotically free field theories. In this Letter, we shall examine a simple asymptotically free field theory, the $O(N)$ Gross-Neveu model 15] of interacting fermions in two spacetime dimensions. In this model mass renormalization is protected by symmetry, so the behavior analogous to (2) is absent. However, it does have logarithmic coupling constant renormalization and we are able to examine its effect in the large $N$ limit which sums all orders in perturbation theory. We shall find that the theory is not renormalizable. As a consequence, if we require that the dynamically generated fermion mass is finite 円, the effective four-fermi coupling is still cutoff dependent and goes to zero as

\footnotetext{
${ }^{1}$ The cutoff dependence of the fermion mass comes from the gap equation. If a different dependence were chosen, there would be tachions in the spectrum of the theory.
} 
the cutoff is removed. It also exhibits an interesting UV/IR duality. In the large momentum limit it has the momentum dependence

$$
\lambda_{e f f}(p)=\frac{8 \pi}{\ln \frac{\Lambda^{2} p^{2}}{M^{4}}}
$$

where $M$ is the dynamically generated fermion mass. There is a low-energy mirror of this behavior in the infrared, for momenta in the range $1 /(\theta \Lambda)^{2}<<$ $p^{2}<<1 /(\theta M)^{2}$,

$$
\lambda_{e f f}(p)=\frac{8 \pi}{\ln \left(\Lambda^{2}(\theta p)^{2}\right)}
$$

This is an example of a field theory that is perfectly renormalizable and nontrivial on a commutative space and is not renormalizable and has trivial correlators in the infinite cutoff limit on a non-commutative space. It can only be non-trivial if the coupling is kept finite. This is in line with suggestions that non-commutative field theories are only well-defined when there is a finite cutoff in both the UV and IR 16.

There is a renormalizable double-scaling limit of the theory that can be taken and in which the cut-off dependence is removed. In this limit, $\theta \rightarrow 0$ and $\Lambda \rightarrow \infty$ holding $\theta \Lambda$ fixed. Space-time is non-commutative only on distance scales of order the UV cutoff. We shall find that, nevertheless, the theory in this limit exhibits a behavior which is quite different from its commutative analog.

2. The Euclidean action of the non-commutative Gross-Neveu model is

$$
S[\psi]=-\int d^{2} x\left\{\frac{1}{2} \sum_{j=1}^{N} \bar{\psi}^{j} \gamma \cdot \partial \psi^{j}+\frac{\lambda}{8 N} \sum_{i j=1}^{N} \bar{\psi}^{i} * \psi^{i} * \bar{\psi}^{j} * \psi^{j}\right\}
$$

Here $\psi^{i}$ are $\mathrm{N} 2$-component Majorana fermions. They obey the constraint $\psi=$ $C \psi^{*}$ with $C$ the charge conjugation matrix. We use Majorana, rather than Dirac fermions because in the latter case, corrections to the four fermion interaction are not sensitive to the non-commutativity in the leading order in $1 / \mathrm{N}$.

The kinetic term in (ब) has $O(N)_{L} \times O(N)_{R}$ chiral symmetry. The interaction term breaks this to a diagonal $O(N)$. There is also a discrete chiral symmetry, $\psi \rightarrow \gamma^{5} \psi$ with $\gamma^{5}=i \gamma^{1} \gamma^{2}$. The condensate $\langle\bar{\psi} \psi\rangle$ is an order parameter for breaking of this symmetry. If it is non-zero, the fermions are massive. All products in (4) are $*$ products, as defined in (11). When we set total derivative

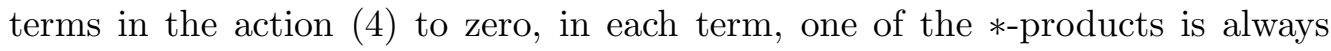
equal to an ordinary product. For this reason only the ordinary product occurs in quadratic terms. The quartic term can be written as $\int(\bar{\psi} * \psi)^{2}$. We shall use this fact later when we introduce an auxiliary field. Note that in two dimensions non-commutativity does not break Lorenz invariance, $\theta_{\mu \nu}=\theta \cdot \epsilon_{\mu \nu}$. Also, in Minkowski space, it would not be possible to set $\theta^{0 i}$ to zero, so a $1+1$-dimensional noncommutative theory may not be a well-defined Hamiltonian system. Here we take the philosophy that (国) does define a statistical model where correlators can be computed and where issues such as renormability, which do occur in other more physical models can be addresed. 
This model is explicitly solvable in the infinite $N$ limit. The commutative version is asymptotically free. The effective four-fermion coupling decreases with increasing momentum transfer and increases with decreasing momentum, running to strong coupling in the infrared. This running is cut off by spontaneous generation of the fermion mass. The result is a non-trivial, interacting field theory with a dynamically generated mass scale. This is prototypical of some of the behavior which is thought to occur in other asymptotically free theories such as four dimensional Yang-Mills theory.

In order to solve the large $N$ limit of the model (4), it is convenient to introduce an auxiliary field so that the action is

$$
S[\psi, \phi]=\int d^{2} x\left\{-\frac{1}{2} \sum_{j=1}^{N} \bar{\psi}^{j}(\gamma \cdot \partial+* \phi *) \psi^{j}+\frac{N}{2 \lambda} \phi^{2}\right\}
$$

The original action (雨) is re-obtained by integrating out $\phi$ in the partition function, $Z=\int[d \psi d \phi] \exp (-S[\psi, \phi])$. Instead, we integrate out $\psi$ to get the non-local scalar field theory with action

$$
S[\phi]=-\frac{N}{2} \operatorname{Tr} \ln (\gamma \cdot \partial+* \phi *)+\int \frac{N}{2 \lambda} \phi^{2}
$$

where $* \phi *$ denotes multiplication using the $*$-product. In the large $N$ limit, the remaining functional integral can be evaluated by saddle point approximation. For this, we must find a minimum of (6) as a functional of $\phi$. We will restrict our search for minima to those which give translation invariant ground states, i.e. to where the function $\phi$ which minimizes (6) is a constant. At this point, we do not know whether there are translation non-invariant solutions which are have smaller action than the translation invariant solution that we find. We will not address this issue in this Letter. Since we find no tachyons in the spectrum, the solution which we consider is at least a local minumum. If $\phi=M$ is a constant, we can readily evaluate (6). Divided by the space-time volume, it is the effective potential

$$
V_{\text {eff }}=-\frac{N}{8 \pi}\left(M^{2} \ln \frac{\Lambda^{2}}{M^{2}}+M^{2}\right)+\frac{N}{2 \lambda} M^{2}
$$

which must be minimized in order to find the physical value of $M$. It always has a minimum for non-zero $M$ which occurs when

$$
\frac{1}{\lambda}=\frac{1}{4 \pi} \ln \frac{\Lambda^{2}}{M^{2}}
$$

This equation is dimensional transmutation: the bare dimensionless coupling $\lambda$ and UV cutoff are traded for a dimensional parameter, $M$, the dynamically generated fermion mass.

The effective four-fermion coupling is determined by the quadratic fluctuations of $\phi$. Consider $\phi=M+\delta \phi$ in (6). Since $\delta \phi$ is not a constant, we must be careful to take into account the $*$-product in the determinant. The determinant is defined by the expression 


$$
-\frac{1}{2} \ln \operatorname{Tr}(\gamma \cdot \partial+* \phi *)=\sum_{n=1}^{\infty} \frac{1}{n !} \int \delta \phi\left(x_{1}\right) \ldots \delta \phi\left(x_{n}\right) \tau\left(x_{1}, \ldots, x_{n}\right)
$$

where

$$
\tau\left(x_{1}, \ldots, x_{n}\right)=-\left(\frac{1}{2}\right)^{n}\left\langle\bar{\psi}\left(x_{1}\right) * \psi\left(x_{1}\right) \ldots \bar{\psi}\left(x_{n}\right) * \psi\left(x_{n}\right)\right\rangle_{0}^{\mathrm{conn} .}
$$

The expectation values are taken with respect to free fermions with mass $M$. Of particular interest is the quadratic term with $\tau\left(x_{1}, x_{2}\right)=\int \frac{d^{2} q}{(2 \pi)^{2}} \tau(q) e^{i q \cdot\left(x_{1}-x_{2}\right)}$. It gets a contribution from a planar and a non-planar diagram and thus depends on the non-commutativity parameter. The planar diagram contributes

$$
\tau_{1}(q)=-\frac{1}{4 \pi}\left(\ln \frac{\Lambda e^{1-\gamma}}{M}-\frac{\sqrt{1+\frac{q^{2}}{4 M^{2}}}}{\frac{q}{2 M}} \ln \left(\sqrt{1+\frac{q^{2}}{4 M^{2}}}+\frac{q}{2 M}\right)\right)
$$

where $\gamma$ is Euler's constant. Here, we have used the same regularization as in 8 and [9]. The non-planar contribution is

$$
\begin{gathered}
\tau_{2}(q)=-\frac{1}{4 \pi} K_{0}\left(2 M \sqrt{\left[\theta^{2} q^{2} / 4+1 / \Lambda^{2}\right]}\right)+ \\
+\frac{1}{4 \pi}\left(M^{2}+\frac{q^{2}}{4}\right) \int_{0}^{1} d \alpha \int_{0}^{\infty} d \rho \exp \left\{-\rho\left(M^{2}+\alpha(1-\alpha) q^{2}\right)-\frac{(\theta q)^{2}}{4 \rho}\right\} .
\end{gathered}
$$

Here $K_{0}(z)$ is the modified Bessel function. The effective four point coupling of the fermions with momentum transfer $q$ is

$$
\lambda_{\text {eff }}(q)=\frac{1}{\frac{1}{\lambda}+\tau_{1}(q)+\tau_{2}(q)}
$$

When we substitute the cut-uff-dependent expression (8) for $1 / \lambda$ into (13), the UV cutoff dependence does not cancel. If $q>1 / \theta \Lambda$, the effective coupling $\lambda_{\text {eff }}(q)$ goes to zero as $\Lambda$ is taken to infinity.

3. Let us find UV behaviour of (13). In the limit when $q^{2}>>4 M^{2}, q^{2}>>$ $1 /(\theta M)^{2}$ (we always assume that $q^{2}<<\Lambda^{2}$ and $M^{2}<<\Lambda^{2}$ ) we have

$$
\tau_{1}(q) \approx-\frac{1}{8 \pi} \ln \frac{\Lambda^{2}}{q^{2}} \quad \text { and } \quad \tau_{2}(q) \sim e^{-\theta M q}
$$

Thus

$$
\lambda_{\mathrm{eff}}(q) \approx \frac{1}{\frac{1}{\lambda}-\frac{1}{8 \pi} \ln \frac{\Lambda^{2}}{q^{2}}}=\frac{8 \pi}{\ln \frac{\Lambda^{2} q^{2}}{M^{4}}},
$$

where $\lambda$ is eliminated using (8).

On the other hand, when $q^{2}<<1 /\left(\theta^{2} M^{2}\right), q^{2}<<4 M^{2}$ and $q^{2}>>1 /(\theta \Lambda)^{2}$ we can approximate the above expressions by 


$$
\tau_{1}(q) \approx-\frac{1}{8 \pi} \ln \frac{\Lambda^{2}}{M^{2}} \quad \text { and } \quad \tau_{2}(q) \approx-\frac{1}{8 \pi} \ln \left(\theta^{2} q^{2} M^{2}\right)
$$

Hence

$$
\lambda_{\mathrm{eff}}(q) \approx \frac{1}{\frac{1}{\lambda}-\frac{1}{8 \pi} \ln \frac{\Lambda^{2}}{M^{2}}-\frac{1}{8 \pi} \ln \left(\theta^{2} q^{2} M^{2}\right)}=\frac{8 \pi}{\ln \left(\Lambda^{2} \theta^{2} q^{2}\right)} .
$$

For momenta above $q \sim 1 / \theta \Lambda$ the last expression depends on the cutoff and for finite, nonzero momentum it goes to zero as the cutoff goes to infinity.

Quite interesting things happen in the double scaling limit when $\Lambda \rightarrow \infty$ and $\theta \rightarrow 0$ so that $\Lambda \theta=C / M$ with an arbitrary constant $C$. The physical meaning of this limit is that one "regularizes" the ordinary Gross-Neveu model by a non-commutative one at the cutoff scale. In this limit we can obtain an exact expression:

$$
\lambda_{\text {eff }}(q)=\frac{4 \pi}{\frac{1}{2} \ln \left(1+C^{2} q^{2} / M^{2}\right)+\frac{\sqrt{1+\frac{q^{2}}{4 M^{2}}}}{\frac{q}{2 M}} \ln \left(\sqrt{1+\frac{q^{2}}{4 M^{2}}}+\frac{q}{2 M}\right)} .
$$

The second term in the denominator has a squareroot cut starting from $q=2 M i$ in the complex $q$ plane, which corresponds to a pair production of fermions. This is the same as what occurs in the commutative Gross-Neveu model. What is new and interesting is the first term. It has a logariphmyc cut starting from $q=$ $i M / C$. This cut is absent in the commutative model. It probably corresponds to a creation of pairs of some non-local solitons present in the non-commutative theory, which survive the double scaling limit. We see that the limits $\Lambda \rightarrow \infty$ and $\theta \rightarrow 0$ do not commute and even in the case when non-commutativity is relevant at the cutoff scale it still modifies the behaviour of the theory at any energy scale.

4. We have found that if we allow $\Lambda$ to go to infinity with fixed $\theta$, this particular asymptotically free theory is trivial. We argue that this is a generic feature of non-commutative field theories. In fact, consider the following general physical arguments. An excitation with large momentum $p_{x}$ in a non-commutative theory has uncertainty in its position along the momentum $\Delta x \sim 1 / p_{x}$ which is very small. Hence, taking into account that $x$ and $y$ coordinates do not commute, we see that the uncertainty in $y$ is very big. This mixes IR and UV limits in the sense that IR effects modify UV limit and vise versa [17]. As we see from our example, this mixing is generally model independent and generic for theories with a dynamically generated mass gap. Taken to its extreme conclusion it would imply that all non-commutative theories with asymptotic freedom are trivial and therefore, in particular, the limit of open string theory in a constant $B$-field is a trivial theory unless the ultraviolet cutoff $1 / \alpha^{\prime}$ is kept finite. 
It is straightforward to generalize our results to the $2+1$ dimensional GrossNeveu model and to $2+1$ and 3+1-dimensional $O(N)$ vector model with quartic interactions of scalars and space-space non-commutativity. There, the conclusions are even more drastic than in the present two dimensional case.

This work is supported in part by NSERC of Canada. E.T.A. was supported by a NATO Science Fellowship and grants INTAS-97-01-03 and RFBR 98-0216575. We thank Kostya Zarembo for valuable discussions.

\section{References}

[1] P. Ho and Y. Wu, "Noncommutative geometry and D-branes," Phys. Lett. B398, 52 (1997) hep-th/9611233.

[2] A. Connes, M. R. Douglas and A. Schwarz, "Noncommutative geometry and matrix theory: Compactification on tori," JHEP 9802, 003 (1998) hepth/9711162.

[3] C. Chu and P. Ho, "Constrained quantization of open string in background B field and noncommutative D-brane," Nucl. Phys. B568, 447 (2000) hepth/9906192.

[4] N. Seiberg and E. Witten, "String theory and noncommutative geometry," JHEP 9909, 032 (1999) hep-th/9908142].

[5] T. Lee, "Canonical quantization of open string and noncommutative geometry," Phys. Rev. D62, 024022 (2000) [hep-th/9911140].

[6] M. Laidlaw, "Noncommutative geometry from string theory: Annulus corrections," hep-th/0009068.

[7] J. Gomis and T. Mehen, "Space-time noncommutative field theories and unitarity," hep-th/0005129.

[8] S. Minwalla, M. Van Raamsdonk and N. Seiberg, "Noncommutative perturbative dynamics," hep-th/9912072.

[9] M. Van Raamsdonk and N. Seiberg, "Comments on noncommutative perturbative dynamics," JHEP 0003, 035 (2000) hep-th/0002186.

[10] T. Filk, "Divergencies in a field theory on quantum space," Phys. Lett. B376, 53 (1996).

[11] S. S. Gubser and S. L. Sondhi, "Phase structure of non-commutative scalar field theories," hep-th/0006119.

[12] I. Chepelev and R. Roiban, "Renormalization of quantum field theories on noncommutative $\mathrm{R}^{* *}$ d. I: Scalars," JHEP 0005, 037 (2000) hepth/9911098.

[13] I. Chepelev and R. Roiban, "Convergence theorem for non-commutative Feynman graphs and renormalization," hep-th/0008090.

[14] A. Micu and M.Sheikh-Jabbari, "Non-commutative $\Phi^{4}$ theory at two loops", hep-th/0008057.

[15] D. J. Gross and A. Neveu, "Dynamical Symmetry Breaking In Asymptotically Free Field Theories," Phys. Rev. D10, 3235 (1974).

[16] J. Ambjorn, Y. M. Makeenko, J. Nishimura and R. J. Szabo, "Nonperturbative dynamics of noncommutative gauge theory," Phys. Lett. B480, 399 (2000) hep-th/0002158. 
[17] A. Matusis, L. Susskind and N. Toumbas, "The IR/UV connection in the non-commutative gauge theories", hep-th/0002075. 